\title{
Gastroduodenal Mucosal Damage With Salsalate Versus Aspirin: Results of Experimental Models and Endoscopic Studies in Humans
}

\author{
By James M. Scheiman and Grace H. Elta
}

Animal models have identified multiple mechanisms of aspirin toxicity. Aspirin inhibits cyclooxygenase in the gastroduodenal mucosa leading to a decrease in endogenous prostaglandins. Prostaglandin mediated mucus and bicarbonate secretion, epithelial hydrophobicity, blood flow. and cellular proliferation are all decreased. Salicylates may cause direct cellular toxicity via inhibition of energy metabolism and membrane transport properties. Salicylate preparations have been designed to decrease gastroduodenal absorption. Endoscopic studies in humans have confirmed that buffering of aspirin does not ameliorate damage, but enteric coating does. Salicylsalicylic acid (salsalate) is an effective antirheumatic drug that bypasses gastric absorption and also avoids cyclooxygenase inhibition. In a randomized, single-blind, endoscopic comparison of salsalate versus enteric-coated aspirin, significantly less gastroduodenal damage was observed in volunteers after salsalate administration compared to enteric-coated aspirin. An endoscopic study in rheumatoid arthritics also confirmed the ability of salsalate to spare gastroduodenal mucosa when compared to naproxen administration. Salsalate may cause less gastroduodenal damage than entericcoated aspirin based on the results of animal models and endoscopic studies in humans.

(C) 1990 by W.B. Saunders Company.

INDEX WORDS: Salicylates; gastroduodenal damage; animal models; endoscopic studies.

QALICYLATES and other nonsteroidal anti$\checkmark$ inflammatory drugs (NSAIDs) are some of the most commonly used drugs in clinical practice. Epidemiological studies have estimated that they are prescribed in quantities sufficient to treat three million people on a daily basis, suggesting as many as $1.2 \%$ of the United States population may use these agents daily. In 1983 over half the physician visits for arthritis involved prescriptions for NSAIDs. ${ }^{1}$

While these agents are very effective in the care of patients with painful musculoskeletal disorders, their gastrointestinal (GI) side effects remain a vexing clinical problem. Given the large number of patients exposed to these drugs,
NSAID-induced GI toxicity may represent the most frequent drug side effect in the US. ${ }^{2}$ This situation has generated interest in the development of strategies designed to limit gastroduodenal injury caused by chronic NSAID use. Concomitant use of conventional antiulcer therapy for both prevention of upper GI tract ulceration and treatment of NSAID-induced damage while the NSAID is continued, has shown mixed and largely disappointing results. ${ }^{3}$ Recently it has been suggested that oral prostaglandins may have a role in the prophylaxis of gastroduodenal damage caused by NSAIDs. ${ }^{4}$ Unfortunately, they are costly and have significant side effects.

An alternative to the administration of an additional drug, such as a prostaglandin, is to utilize an NSAID which spares the upper GI tract. Salicylsalicylic acid (salsalate), a salicylate ester of salicylic acid, was originally developed as a safer, nonacetylated alternative to aspirin (acetylsalicylic acid). ${ }^{5}$ In this review we will discuss the mechanisms of salicylate-induced gastroduodenal damage in animal models and the results of endoscopic studies in humans that suggest salsalate has potential as a gastroduodenal sparing agent.

\section{MUCOSAL INJURY BY SALICYLATES IN ANIMAL MODELS}

Aspirin is absorbed passively in the upper GI tract. This absorption is dependent on its concentration and its lipid solubility at acid $\mathrm{pH}$ (the $\mathrm{pKa}$ of aspirin is 3.5 ). The compound is more

From the Department of Internal Medicine, Division of Gastroenterology, University of Michigan Medical School, and Veterans Administration Medical Center, Ann Arhor. $M I$.

James M. Scheiman, MD: Instructor in Internal Medicine; University of Michigan Medical School; Grace H. Elta; MD: Assistant Professor of Internal Medicine, University of Michigan Medical School.

Address reprint requests to James $M$. Scheiman, $M D, G I$ Section (ll1D), VA Medical Center, 2215 Fuller Rd, Ann Arbor, MI 48105.

(c) 1990 by W.B. Saunders Company

0049-0172/90/2002-0007\$5.00/0 
than $50 \%$ nonionized in gastric or duodenal fluid with a $\mathrm{pH}$ below 3.5 and rapid absorption occurs. Once absorbed across the cell membrane, the higher intracellular $\mathrm{pH}$ favors dissociation to the ionized form, leading to accumulation and high local cellular concentrations of the drug. When the aspirin is completely absorbed, it is hydrolyzed to salicylic acid by the action of intestinal, hepatic, and plasma esterases. The toxicity of acetylsalicylic acid depends upon both a local toxic effect and the systemic effect of the drug. Animal models have offered insight into the mechanisms of aspirin induced-toxicity. Depending upon the model studied, and the criterion to measure damage, some animal models show greater toxicity with aspirin than salicylic acid.

\section{Cyclooxygenase Independent Mechanisms}

Acetylsalicylic acid inhibits mucosal cyclooxygenase, thereby decreasing levels of endogenous prostaglandins. In contrast, salicylate is a weak inhibitor of the enzyme. ${ }^{6}$ In addition to this prostaglandin mediated effect, salicylates can have direct cellular toxicity independent of cyclooxygenase inhibition.

Pioneering work by Davenport demonstrated that the gastric mucosal barrier, the unique ability of the gastric surface epithelium to survive in the hostile acidic milieu of the stomach, is lost after exposure to either aspirin or salicylic acid. ${ }^{7}$ Both drugs increase proton flux into the mucosa and cause a drop in potential difference, a sensitive measure of epithelial integrity. Other investigators have confirmed this decrease in mucosal potential difference, with increased cation flux after exposure to both aspirin and salicylic acid, yet only aspirin caused a dramatic reduction in mucosal prostaglandin content. ${ }^{8}$ The back diffusion of protons leads to cellular acidification and altered cellular metabolism.

An additional mechanism of salicylate induced damage independent of cyclooxygenase inhibition is the uncoupling of oxidative phosphorylation by intracellular salicylates. This results in depletion of cellular adenosine triphosphate (ATP) levels and inhibition of active ion transport essential to maintain cellular integrity. This effect on energy metabolism is a major direct toxic effect of salicylate. ${ }^{9}$

Although salicylate has direct toxic effects on gastric epithelia, a number of studies have dem- onstrated that sodium salicylate administration fails to injure gastric mucosa. ${ }^{6,10}$ Investigators have inferred from these data that cyclooxygenase inhibition is the most important mechanism of gastrointestinal injury by salicylates. Recent in vitro studies suggest that salicylic acid and aspirin can damage mucosa equally, but only after the gastric luminal $\mathrm{pH}$ has been lowered to 1.0. ${ }^{11,12}$ These damaging effects occur with either oral or parenteral administration. Because parenteral salicylic acid inhibits acid secretion to a much greater extent than acetylsalicylic acid, the lack of gastric damage produced by salicylic acid in previous studies may have been caused by insufficient luminal acidification. When luminal $\mathrm{pH}$ is decreased, salicylate appears to accumulate in the epithelium and diminish mucosal resistance to proton flux. To further elucidate the role of cyclooxygenase inhibition in the genesis of mucosal toxicity, investigators attempt to reverse or prevent damage by exogenous administration of prostaglandins. Partial amelioration of the aspirin injury was observed after administration of prostaglandin $E_{2}\left(P_{2}\right)$ but no effect on the salicylate injury was seen. ${ }^{12}$ The partial protective effect of $\mathrm{PGE}_{2}$ points to the complex mechanisms of aspirin injury and suggests that additional mechanisms of damage related to cyclooxygenase inhibition may be important in the intact animal. An understanding of the effects of cyclooxygenase inhibition on the components of gastroduodenal defense is required for further understanding of the mechanisms of aspirin toxicity.

\section{Cyclooxygenase Dependent Mechanisms}

Robert first described the ability of prostaglandins, independent of their inhibition of acid secretion, to protect the gastric mucosa against a variety of noxious agents that produce tissue damage and cellular necrosis. ${ }^{13}$ This was called "cytoprotection." This observation prompted many investigations into the role of endogenous prostaglandins in the maintenance of upper GI mucosal integrity. The major prostaglandins synthesized by the action of cyclooxygenase on membrane arachidonic acid within gastrointestinal mucosa include $\mathrm{PGE}_{2}, \mathrm{PGI}_{2}, \mathrm{PGD}_{2}$, and $\mathrm{PGF}_{2} \alpha$. To investigate the physiological role of endogenous prostaglandins, most studies have examined the effect of aspirin or other NSAID 
cyclooxygenase inhibitors on a parametcr important in mucosal defense.

Recently, Redfern and Feldman demonstrated that active immunization of rabbits, with the principal endogenous prostaglandins of gastrointestinal mucosa, induced upper GI tract ulceration. ${ }^{14}$ These lesions resemble both human stress ulcers and the erosive gastric disease seen with NSAID administration. The authors postulated that prostaglandin antibodies led to a decrease in effective tissue content of prostaglandins, resulting in either an increase in gastric acid secretion or a decrement in mucosal defense. Active immunization of dogs with $\mathrm{PGE}_{2}$ did not increase acid secretion. Therefore, endogenous prostaglandins appear to have a greater role in the maintenance of mucosal defensive factors compared to their acid inhibitory effect. ${ }^{15}$

\section{Prostaglandins and Mucosal Defense}

Critical to the maintenance of gastroduodenal integrity is the presence of a mucous gel into which bicarbonate is secreted, forming an unstirred water layer with a protective $\mathrm{pH}$ gradient adjacent to the mucosa. ${ }^{16} \mathrm{~A} \mathrm{pH}$ gradient from $\mathrm{pH} 2$ in the lumen to approximately $\mathrm{pH} 7$ at the cell membrane has been confirmed in animals by passing a $\mathrm{pH}$ probe through the mucous gel. ${ }^{17}$ Recent work using in vivo microscopy has demonstrated that this gradient is preserved through active expulsion of acid from the base of the gastric gland with preservation of the mucous gel and its $\mathrm{pH}$ gradient. ${ }^{18}$ Prostaglandin administration has been shown to enhance the release of both mucus and bicarbonate, while cyclooxygenase inhibition diminishes them. Aspirin and other NSAIDs significantly inhibit basal bicarbonate secretion from an amphibian model in vitro, an effect reversed by administration of synthetic PGE $_{2}{ }^{19}$ This observation has been confirmed in humans by Isenberg who found that indomethacin inhibited duodenal bicarbonate and $\mathrm{PGE}_{2}$ production. ${ }^{20}$ To our knowledge, the effects of salicylates other than aspirin on basal bicarbonate secretion have not been studied.

Prostaglandins have an important role in the maintenance of mucus secretion but seem to have little role in the synthesis of glycoprotein. ${ }^{21}$ Both $\mathrm{PGE}_{2}$ and $\mathrm{PGF}_{2}$ increase secretion of glycoprotein, while indomethacin has an inhibitory effect. These investigators found no effect of prostaglan- dins on mucin synthesis, while others suggest that aspirin can qualitatively alter mucus, such as inhibition of sulfate incorporation. ${ }^{22}$ Salicylic acid exposure appears to have a much weaker effect. $^{22}$ Another qualitative deficiency of gastric mucus caused by aspirin exposure is increased sensitivity to pepsin leading to the formation of smaller and less viscous gel which is poorly resistant to $\mathrm{H}^{+}$penetration. ${ }^{23}$

An additional important defensive factor that appears prostaglandin dependent is the maintenance of surface hydrophobicity of the gastric epithelium. The presence of saturated phospholipids, which have surface-active properties, appear to cause the gastric surface to be uniquely hydrophobic and thus repel water and acid. This effect can be measured by the formation of a contact angle between a drop of water and the gastric surface. Administration of aspirin leads to loss of surface hydrophobicity and decreased transmucosal potential difference, ${ }^{24}$ with prostaglandin administration restoring the surface hydrophobicity lost after aspirin exposure. ${ }^{25}$ The effects of nonaspirin salicylates on surface hydrophobicity is unknown.

Additional cyclooxygenase dependent effects of aspirin include a decrease in mucosal blood flow due to the loss of tonic vasodilatory prostaglandins. Microcirculatory studies have demonstrated arteriolar constriction and thrombus formation after luminal aspirin exposure. ${ }^{26}$ These effects may contribute to ischemia causing mucosal injury and intramucosal hemorrhage. Prostaglandins also have been shown to have trophic effects on gastric mucosa. Inhibition of proliferation may be an additional mechanism of aspirin toxicity. ${ }^{9}$

In summary, the toxicity of aspirin for the gastrointestinal epithelium is a combination of the direct effects of salicylate on cellular function and depletion of prostaglandin-dependent mucosal defensive factors (Table 1). Most models of mucosal injury demonstrate a correlation between mucosal inhibition of cyclooxygenase and observed damage. Salicylic acid has little effect on the enzyme, less interference with prostaglandin mediated mucosal defense, and thus less injury is generally observed. We turn now to endoscopic studies in humans to determine if the differences noted in animal models can be used for clinical advantage. 
Table 1: Gastroduodenal Toxicity of Aspirin: Cellular Mechanisms

Events related to cyclooxygenase inhibition-Loss of prostaglandin mediated

Mucus secretion

Viscosity and acid resistance of mucus

Bicarbonate secretion

Hydrophobicity of the epithelial surface

Blood flow

Cellular proliferation

Events related to the direct cellular toxicity of salicylate

Increased permeability - cation flux

Decreases in transmucosal membrane potential Inhibition of oxidative phosphorylation Inhibition of membrane transport properties

\section{ENDOSCOPIC STUDIES COMPARING SALICYLATES IN HUMANS}

Because the toxic effects of aspirin relate to both its local concentration and systemic distribution, attempts to lessen direct gastroduodenal absorption have been used. The antacid amelioration of aspirin-induced gastric damage suggests that decreases in local toxicity could be reduced if absorption was delayed. ${ }^{27}$ The use of a "prodrug," a drug given in an inactive form that requires metabolic activation after absorption, is an attempt to lessen the direct gastric absorption of active drug. An endoscopic study with sulindac sulfoxide (the prodrug converted to the cyclooxygenase inhibitor sulindac sulfide) demonstrated markedly less damage compared with aspirin administration, while no difference was seen between the prodrug and the active moiety, perhaps because both were insoluble in gastric juice. ${ }^{28}$ Although little damage was seen after acute administration of sulindac to normal volunteers, a recent large epidemiological study has indicated that sulindac has no advantage over other NSAIDs for risk of upper GI bleeding. ${ }^{29}$ These results suggest that prevention of gastroduodenal absorption is only one aspect in the prevention of NSAID toxicity, and that cyclooxygenase inhibition from a systemically distributed drug is important. The observation that administration of an oral prostaglandin is effective in the prevention of NSAID damage also underscores the importance of endogenous prostaglandins in the maintenance of mucosal integrity. Salicylsalicylic acid (salsalate) is a drug that bypasses gastric absorption (it is insoluble in acid and is hydrolyzed to two molecules of salicylic acid at $\geq 7 \mathrm{pH}$ ) and a drug that does not significantly inhibit cyclooxygenase. Entericcoated aspirin bypasses gastric absorption, but inhibits cyclooxygenase.

Investigations of endoscopic damage after administration of aspirin preparations suggest that buffered preparations offer no advantage over unbuffered preparations. In a review of many studies of acute drug administration in normal subjects, Lanza found that neither Bufferin (Bristol-Meyers; New York, NY) or Ascriptin A-D (W.H. Rorer, Inc; Fort Washington, PA) caused less endoscopic damage than unbuffered acetylsalicylic acid at 2,600 and 3,900 mg/d. ${ }^{30}$ Entericcoated aspirin, however, led to significantly less damage than aspirin. Hoftiezer et al performed a similar endoscopic comparison between plain and enteric-coated aspirin and observed similar protective properties for the enteric-coated preparation. ${ }^{31}$

We studied salsalate, a drug that does not inhibit endogenous prostaglandins and bypasses gastric absorption. This theoretically could represent a therapeutic advantage over enteric-coated aspirin. We performed a randomized, singleblind, crossover, endoscopic comparison of the two drugs after one week of administration in normal volunteers. ${ }^{32}$ Subjects were given salsalate (Disalcid, 3M Pharmaceuticals, St Paul, MN) $1,500 \mathrm{mg} / \mathrm{d}$ (two $750 \mathrm{mg}$ tablets twice a day) or enteric-coated aspirin (Ecotrin, SmithKline Beckman, Philadelphia, PA) $650 \mathrm{mg}$ (two $325 \mathrm{mg}$ tablets) four times a day. The subjects had endoscopy performed prior to drug administration and after 6 days of drug ingestion. After a 1 week "washout" period, the subjects began treatment with the other study drug. We quantitated gastroduodenal damage using an endoscopy rating scale as shown in Table 2 . The results of the endoscopic scores after drug administration, the order of drug dosing, and the posttreatment salicylate levels are summarized in Table 3 . Only a single subject treated with salsalate had any evidence of gastroduodenal damage (grade 1) while 6 of the 10 subjects who received enteric-coated aspirin developed significant damage (grade 2 in one subject and grade 3 in the remainder, including a duodenal ulcer in one patient). Salsalate caused significantly less gas- 
Table 2: Rating of Gastroduodenal Damage

\begin{tabular}{|cc|}
\hline Score & Endoscopic Findings \\
\hline 0 & $\begin{array}{c}\text { Normal } \\
\text { Less than } 3 \text { areas of mucosal } \\
\text { hemorrhage or erosion } \\
\text { 3-10 areas of mucosal hemor- } \\
\text { rhage or erosion } \\
\text { Widespread involvement or } \\
\text { greater than 10 areas of ero- } \\
\text { sions with active bleeding }\end{array}$ \\
\hline
\end{tabular}

troduodenal damage than enteric-coated aspirin when analyzed by the Wilcoxon signed rank test $(P=.01)$, a difference that was maintained when the patient with a baseline antral endoscopic abnormality was excluded from analysis. Gastrointestinal symptoms were mild and not significantly different in the two groups. Mean fasting salicylate levels after 6 days of treatment with salsalate and enteric-coated aspirin were $18.1 \mathrm{mg} / \mathrm{dL}$ and $11.2 \mathrm{mg} / \mathrm{dL}$ respectively. There was no correlation between the serum salicylate level and the development of gastroduodenal damage.

A recent endoscopic study in rheumatoid arthritics has confirmed salsalate's ability to spare the gastroduodenal mucosa from injury while remaining an effective antirheumatic agent. ${ }^{33}$ In this study, eligible patients were randomized to salsalatc or naproxen (Naprosyn, Syntex Laboratories, Palo Alto, CA) for 3 months and gas- troduodenal injury was graded endoscopically after 1 and 3 months. Patients were eligible if they did not have a history of major GI hemorrhage, ulceration larger than $2 \mathrm{~cm}$, or diffuse erosions or ulceration at baseline endoscopy. Patients were given the study drugs without a washout period and the initial study doses were $1,500 \mathrm{mg}$ of salsalate or $375 \mathrm{mg}$ of naproxen twice a day. Doses were titrated to antirheumatic efficacy within the range of 2,000 to $4,000 \mathrm{mg} / \mathrm{d}$ of salsalate and 500 to $1,000 \mathrm{mg} / \mathrm{d}$ naproxen. The distribution of baseline endoscopy scores in the patients who completed the study were not significantly different. The final endoscopy scores were significantly different, with 8 of 21 (38\%) of the naproxen-treated patients developing ulcers (seven patients) or diffuse erosions (one patient) while none of the salsalate patients had either category of lesion $(P=.003$ by the Wilcoxon signed rank test). This study suggests salsalate causes significantly less gastroduodenal toxicity than naproxen after chronic administration to rheumatic patients

The usefulness of endoscopic studies after acute NSAID administration has been questioned because of poor correlation with chronic drug epidemiology. ${ }^{34}$ Also there is poor correlation between acute and chronic studies of antiulcer drugs used for prophylaxis or therapy of NSAID-induced toxicity. ${ }^{35}$ Critics of these endoscopic studies are concerned that acute lesions

Table 3: Endoscopy Sores and Serum Salicylate Levels In 10 Volunteers During Treatment With Salsalate and Enteric-Coated Aspirin*

\begin{tabular}{|c|c|c|c|c|c|}
\hline \multirow[b]{2}{*}{ Subject } & \multicolumn{2}{|c|}{ Salsalate } & \multicolumn{2}{|c|}{ Enteric-Coated Aspirin } & \multirow[b]{2}{*}{$\begin{array}{l}\text { Dosing } \\
\text { Order }\end{array}$} \\
\hline & $\begin{array}{l}\text { Endoscopy } \\
\text { Score }\end{array}$ & $\begin{array}{c}\text { Salicylate } \\
\text { Level }(\mathrm{mg} / \mathrm{dL})\end{array}$ & $\begin{array}{l}\text { Endoscopy } \\
\text { Score }\end{array}$ & $\begin{array}{c}\text { Salicylate } \\
\text { Level (mg/dL) }\end{array}$ & \\
\hline 1 & 0 & 38.8 & 0 & 10.0 & ASA-SAS \\
\hline 2 & 0 & 19.0 & 3 & 15.6 & ASA-SAS \\
\hline 3 & 0 & 10.0 & 2 & 6.6 & ASA-SAS \\
\hline 4 & 0 & 24.7 & 0 & 5.6 & ASA-SAS \\
\hline 5 & 0 & 16.5 & 0 & 8.9 & SAS-ASA \\
\hline 6 & 0 & 17.0 & 3 & 9.6 & SAS-ASA \\
\hline 7 & 1 & 16.3 & 0 & 12.2 & SAS-ASA \\
\hline 8 & 0 & 18.0 & 3 & 15.4 & SAS-ASA \\
\hline 9 & 0 & 6.9 & 3 & 14.1 & ASA-SAS \\
\hline $10^{*}$ & 0 & 13.4 & 3 & 13.8 & ASA-SAS \\
\hline
\end{tabular}

Abbreviations: ASA, aspirin; SAS, salsalate.

* Volunteer 10 had a score of 1 on each baseline endoscopy.

All other baseline scores were 0 . 
such as hemorrhages and erosions do not always correlate with the risk of development of a chronic ulcer or significant GI bleeding. Although studies of longer duration in rheumatic patients are required to confirm the promising results of our endoscopic comparison, salsalate may prove to have significant gastroduodenal sparing properties compared with enteric-coated aspirin. The subjects taking enteric-coated aspirin in our endoscopic study developed more gastroduodenal injury than those in prior reports. $^{30,31}$ These differences likely represent variability from the small number of subjects studied. Endoscopic studies in rheumatic patients suggest that the frequency of gastric, ${ }^{36}$ but not duodenal damage, is reduced with treatment with entcric-coated aspirin. ${ }^{37}$ An additional challenge to the sparing effects of enteric-coated aspirin has recently been made. ${ }^{38}$ Patients who had an NSAID-related ulcer but required chronic antiarthritic therapy were given enteric-coated aspirin in addition to a combination of cimetidine and antacids. None of the patients taking entericcoated aspirin healed on therapy while 15 out of 16 who stopped their NSAIDs healed. Thus, enteric coating of aspirin may afford some gastroduodenal protection, but it is not risk free. We conclude that salsalate may cause less gastroduodenal damage than enteric-coated aspirin based on the results of animal models and our endoscopic comparison.

\section{REFERENCES}

1. Baum C, Kennedy DL: Utilization of anti-inflammatory drugs. Arthritis Rheum 28:686-689, 1984

2. Fries JF, Miller SR, Spitz PW, et al: Toward and epidemiology of gastropathy associated with nonsteroidal anti-inflammatory drug use. Gastroenterology 96:647-655, 1989

3. Graham DY: Prevention of gastroduodenal injury in duced by chronic nonsteroidal anti-inflammatory drug therapy. Gastroenterology 96:675-681, 1989

4. Graham DY, Agrawal NM, Roth SR: Prevention of NSAID-induced gastric ulcer with misoprostol: Multicenter, double-blind, placebo-controlled trial. Lancet 2:1277-1280, 1988

5. Singleton PT: Salsalate: Its role in the management of rheumatic disease. Clin Ther 3:80-101, 1980

6. Whittle BJR, Higgs GA, Eakins KE, et al: Selective inhibition of prostaglandin production in inflammatory exudates and gastric mucosa. Nature 284:271-273, 1980

7. Davenport $\mathbf{H W}$ : Salicylate damage to the gastric mucosal barrier. N Engl J Med 276:1307-1312, 1967

8. Ligumsky M, Grossman MI, Kaufman GL: Endogenous gastric mucosal prostaglandins: Their role in mucosal integrity. Am J Physiol 242:G337-341, 1982

9. Kauffman G: Aspirin-induced gastric mucosal injury: Lessons learned from animal models. Gastroenterology 96 : 606-614, 1989

10. Hausen D, Salmon JA, Whittle BJR: Ulcerogenesis and prostanoid inhibition in the cat gastric antrum induced by parenteral aspirin but not by salicylate. $\mathrm{Br} \mathbf{J}$ Pharmacol 80:602, 1983

11. Fromm D, Kolis M: Effects of sodium salicylate and acetylsalicylic acid on intramural pH and ulceration of rabbit antral mucosa. Surgery $91: 438-447,1982$

12. Rowe $\mathbf{P H}$, Sarlinger MJ, Kasdon $\mathbf{E}$, et al: Parenteral aspirin and sodium salicylate are equally injurious to the rat gastric mucosa. Gastroenterology 93:863-871, 1987

13. Robert A: Cytoprotection by prostaglandins. Gastroenterology 77:761-767, 1979

14. Redfern JS, Feldman M: Role of endogenous prostaglandins in preventing gastrointestinal ulceration; induc- tion of ulcers by antibodies with antibodies to prostaglandins. Gastroenterology 96:596-605, 1989

15. Redfern JS, Blair AJ, Glubb F, et al: Gastroduodenal ulceration following active immunization with prostaglandin $E_{2}$ in dogs: Role of gastric acid secretion. Prostaglandins 34:623-632, 1987

16. Miller TA: Protective effects of prostaglandins against mucosal damage: Current knowledge and proposed mechanisms. Am J Physiol 245:G601-623, 1983

17. Williams SE, Turnberg LA: Demonstration of a $\mathrm{pH}$ gradient across mucus adherent to rabbit gastric mucosa: Evidence for a 'mucus-bicarbonate' barrier. Gut 22:94-96, 1981

18. Holm LP, Flemstrom G: In vivo microscopy of acid transport at the gastric surface. Gastroenterology 96:A215, 1989

19. Garner A, Flemstrom G, Heylings JR: Effects of anti-inflammatory agents and prostaglandins on acid and bicarbonate secretions in the amphibian-isolated gastric mucosa. Gastroenterology 77:451-457, 1979

20. Selling JA, Hogan DL, Aly A, et al: Indomethacin inhibits duodenal mucosal bicarbonate secretion and endogenous prostaglandin $E_{2}$ output in human subjects. Ann Intern Med 106:368-371, 1987

21. Seidler U, Knafla K, Kownatzki R, et al: Effects of endogenous and exogenous prostaglandins on glycoprotein synthesis and secretion in isolated rabbit gastric mucosa. Gastroenterology 95:945-951, 1988

22. Rainsford KD: The effects of aspirin and other nonsteroid anti-inflammatory/analgesic drugs on gastrointestinal mucus glycoprotein biosynthesis in vivo: Relationship to ulcerogenic actions. Biochem Pharmacol 27:877-885, 1978

23. Sarosiek J, Mizuta K, Slomiany A, et al: Effect of acetylsalicylic acid on gastric mucin viscosity, permeability to hydrogen ion, and susceptibility to pepsin. Biochem Pharmacol 35:4291-4295, 1986

24. Goddard PJ, Hills BA, Lichtenberger LM: Does aspirin damage canine gastric mucosa by reducing its surface hydrophobicity? Am J Physiol 252:G421-430, 1987

25. Lichtenberger LM, Richards JE, Hills BA: Effect of 
16,16-dimethyl prostaglandin $E_{2}$ on the surface hydrophobicity of aspirin-treated canine gastric mucosa. Gastroenterology 88:308-314, 1985

26. Kitahora T, Guth PH: Effect of aspirin plus hydrochloric acid on the gastric mucosal microcirculation. Gastroenterology $93: 810-817,1987$

27. Thorsen WB, Jr, Western D, Tanaka Y, et al: Aspirin injury to the gastric mucosa: Gastrocamera observations of the effect of pH. Arch Intern Med 121:499-506, 1968

28. Graham DY, Smith JL, Holmes GI, et al: Nonsteroidal anti-inflammatory effect of sulindac sulfoxide and sulfide on gastric mucosa. Clin Pharmacol Ther 38:65-70, 1985

29. Carson JL, Strom BL, Morse $\mathbf{L}$, et al: The relative gastrointestinal toxicity of the nonsteroidal anti-inflammatory drugs. Arch Intern Med 147:1054-1059, 1987

30. Lanza FL: Endoscopic studies of gastric and duodenal injury after the use of ibuprofen, aspirin, and other nonsteroidal anti-inflammatory agents. Am J Med 77:19-24, 1984 (suppl 1A)

31. Hoftiezier IW, Silvoso GR, Burks M, et al: Comparison of the effects of regular and enteric-coated aspirin on the gastroduodenal mucosa of man. Lancet 2:609-612, 1980

32. Schciman JM, Bchler EM, Bcrardi RR, et al: Salicylsalicylic acid causes less gastroduodenal damage than enteric- coated aspirin: An endoscopic comparison. Dig Dis Sci 34:229-232, 1989

33. Roth $S$, Bennett $R$, Caldron $P$, et al: Reduced risk of NSAID gastropathy (GI mucosal toxicity) with nonacetylated salicylate (salsalate): An endoscopic study. Semin Arthritis Rheum 19:11-19, 1990 (suppl 2)

34. Graham DY, Smith JL: Gastroduodenal complications of chronic NSAID therapy. Am J Gastroenterol 83:10811084,1988

35. McCarthy DM: Nonsteroidal anti-inflammatory drug induced ulcers: Management by traditional therapies. Gastroenterology 96:662-674, 1989

36. Silvoso GR, Ivey KJ, Butt JH, et al: Incidence of gastric lesions in patients with rheumatic disease on chronic aspirin therapy. Ann Intern Med 91:517-520, 1979

37. Lockark OO, Ivey KJ, Butt JH, et al: The prevalence of duodenal lesions in patients with rheumatic disease on chronic aspirin therapy. Gastrointest Endosc 26:5-7, 1980

38. Jaszewski R, Calzada R, Dhar R: Persistence of gastric ulcers caused by plain aspirin or nonsteroidal antiinflammatory agents in patients treated with a combination of Cimetidine, antacids and enteric-coated aspirin. Dig Dis Sci 34:1361-1364, 1989 